\title{
BEST RATIONAL APPROXIMATIONS OF ENTIRE FUNCTIONS WHOSE MACLAURIN SERIES COEFFICIENTS DECREASE RAPIDLY AND SMOOTHLY
}

\author{
BY
}

\author{
A. L. LEVIN AND D. S. LUBINSKY
}

ABSTRACT. Let $f=\sum_{j=0}^{\infty} a_{j} z^{j}$ be an entire function which satisfies

$$
\left|a_{j-1} a_{j+1} / a_{j}^{2}\right| \leqslant \rho^{2}, \quad j=1,2,3, \ldots
$$

where $0<\rho<\rho_{0}$ and $\rho_{0}=0.4559 \ldots$ is the positive root of the equation $2 \sum_{j=1}^{x} \rho^{j^{2}}$ $=1$. Let $r>0$ be fixed. Let $W_{I . M}$ denote the rational function of type $(L, M)$ of best approximation to $f$ in the uniform norm on $|z| \leqslant r$. We show that for any sequence of nonnegative integers $\left\{M_{l}\right\}_{I=1}^{\infty}$ that satisfies $M_{l} \leqslant 10 L, L=1,2,3, \ldots$, the rational approximations $W_{I . M_{l}}$ converge to $f$ throughout $\mathbf{C}$ as $L \rightarrow \infty$. In particular, convergence takes place for the diagonal sequence and for the row sequences of the Walsh array for $f$.

1. Introduction. As far as the authors can determine, $e^{z}$ is the only function for which best rational approximations are known to overconverge throughout $\mathbf{C}$. The known results, all due to Saff $[7,8]$, include the following. Let $r>0$ be fixed. For each pair $(m, n)$ of nonnegative integers, let $W_{m n}$ denote a rational function of type $(m, n)$ of best approximation to the function $e^{z}$ in the uniform norm on $|z| \leqslant r$.

TheOREM A [7]. As $m+n \rightarrow \infty, W_{m n}(z) \rightarrow e^{z}$ uniformly on compact subsets of $\mathbf{C}$.

THEOREM B [8]. Let $\varepsilon_{m n}=m ! n ! /((m+n) !(m+n+1) !)$. Then, for each fixed $n$,

$$
\max _{|z| \leqslant r}\left|e^{z}-W_{m n}(z)\right|=\varepsilon_{m n} r^{m+n+1}(1+o(1)) \quad \text { as } m \rightarrow \infty \text {. }
$$

Recently Trefethen [9], using the method of Braess [2], extended Theorem B to non-row sequences of the Walsh array for $e^{z}$.

THEOREM C [8]. For each fixed $n$,

$$
\lim _{m \rightarrow \infty} \frac{e^{z}-W_{m n}(z)}{(-1)^{n} \varepsilon_{m n} z^{m+n+1}}=1,
$$

uniformly on each compact subset of $|z|>r$.

Received by the editors January 8,1985 .

1980 Mathematics Subject Classification. Primary 41A20, 41A21: Secondary 30E10.

Key words and phrases. Padé table, best rational approximation, Walsh array.

(1986 American Mathematical Society $0002-9947 / 86 \$ 1.00+\$ .25$ per page 
The proof of Saff's theorems, as well as the work of Trefethen and Braess, is based on the fact that analogous results are valid for the Padé approximations to $e^{z}$.

Recently one of the authors $[3,4]$ investigated the Padé tables of entire functions such as

$$
f(z)=\sum_{j=0}^{\infty} a_{j} z^{j}, \quad a_{j} \neq 0, j=0,1,2, \ldots
$$

which satisfy

$$
\left|a_{j-1} a_{j+1} / a_{j}^{2}\right| \leqslant \rho^{2}, \quad j=1,2, \ldots,
$$

where

$$
\rho<\rho_{0},
$$

and $\rho_{0}=0.4559 \ldots$ is the positive root of the equation $2 \sum_{j=1}^{\infty} \rho^{j^{2}}=1$.

It was shown in [3] that the Padé table of $f$ is normal, and that any sequence of Padé approximants with numerator degrees tending to infinity converges to $f$ uniformly on compact subsets of $\mathbf{C}$. These properties of the Padé approximants (together with their additional properties to be established in §2) enable us to use Saff's argument in a modified form to prove results analogous to Theorems A, B and $\mathrm{C}$ for this class of functions.

Throughout this paper we use the following notation. $[L / M]$ will denote the Pade approximant of type $(L, M)$ for $f$, that is, $[L / M]=P_{L M} / Q_{L M}$, where $P_{L M}$ and $Q_{L M}$ are polynomials of degree $\leqslant L, M$ respectively, $Q_{L M}(0)=1$, and $Q_{L . M}(z) f(z)$ $-P_{L M}(z)=O\left(z^{L+M+1}\right)$ formally. For any $r>0, W_{L M}(z)$ will denote a rational function of type $(L, M)$ of best approximation to $f$ in the uniform norm on $|z| \leqslant r$. That is,

$$
\max _{|z| \leqslant r}\left|f(z)-W_{L M}(z)\right| \leqslant \max _{|z| \leqslant r}|f(z)-R(z)|
$$

for every rational function $R$ of type $(L, M)$.

We shall use $\|g\|_{r}$ to denote $\max _{|z| \leqslant r}|g(z)|$. For $L, M \geqslant 0$ we denote by $D(L / M)$ the determinant

$$
D(L / M)=\left|\begin{array}{cccc}
a_{L} & a_{L+1} & \cdots & a_{L+M-1} \\
\vdots & \vdots & & \vdots \\
a_{L-M+1} & a_{L-M+2} & \cdots & a_{L}
\end{array}\right|
$$

(where $a_{j}=0$ if $j<0$ ) and we let

$$
\varepsilon_{L M}=\frac{D((L+1) /(M+1))}{D(L / M)} .
$$

We now formulate our results. 
THEOREM 1. Let $f$ be an entire function that satisfies (1)-(3). Let $r>0$ be fixed. Let $W_{L M}$ be a rational function of type $(L, M)$ of best approximation to $f$ in the uniform norm on $|z| \leqslant r$. Then:

(i) For any sequence of nonnegative integers $\left\{M_{L}\right\}_{L=1}^{\infty}$ that satisfies $M_{L} \leqslant 10 L$, $L=1,2,3, \ldots$, the sequence $\left\{W_{L M_{l}}\right\}_{L=1}^{\infty}$ converges to $f$ uniformly on compact subsets of $\mathbf{C}$.

(ii) If in (2), $\rho<1 / 3$, then the restriction $M_{L} \leqslant 10 L$ can be omitted.

Remarks. 1. The number 10 may be replaced by any positive number < $4\left|\log \rho_{0}\right| / \log \left(3 \rho_{0}\right)=10.0339 \ldots$

2. Note that Saff's Theorem A admits the case $L=$ const, that is the column sequences of the Walsh array for $e^{z}$ also converge to $e^{z}$. For our class of functions the analogous result is not true. Indeed, any entire function $f$ that satisfies (1), (2) is of order 0 and consequently (since it is not rational) has infinitely many zeroes in $\mathbf{C}$. From Hurwitz' theorem follows that no sequence of rational functions with fixed degree of the numerator can converge to $f$ throughout $\mathbf{C}$.

THEOREM 2. With the notation of Theorem 1 and under the restriction $M_{L} \leqslant 1.4 L$, $L=1,2, \ldots$, we have

(i) $\lim _{L \rightarrow \infty}\left\|f-W_{L M_{l}}\right\|_{r} /\left(\left|\varepsilon_{L M_{l}}\right| r^{L+M_{L}+1}\right)=1$,

(ii) $\lim _{L \rightarrow \infty}\left(f(z)-W_{L M_{L}}(z)\right)(-1)^{M} /\left(\varepsilon_{L M_{l}} z^{L+M_{l .}+1}\right)=1$, uniformly on compact subsets of $|z|>r$.

Remark. The number 1.4 may be replaced by any positive number < $2\left|\log \rho_{0}\right| / \log 3=1.42995 \ldots$

2. Estimations for Padé approximants. We first establish some simple properties of the Maclaurin coefficients of $f$, which follow from condition (2). This condition can be rewritten in either of two forms:

$$
\begin{array}{ll}
\left|a_{j+1} / a_{j}\right| \leqslant \rho^{2}\left|a_{j} / a_{j-1}\right|, & j=1,2,3, \ldots, \\
\left|a_{j-1} / a_{j}\right| \leqslant \rho^{2}\left|a_{j} / a_{j+1}\right|, & j=1,2,3, \ldots
\end{array}
$$

From (6) it follows by induction that

$$
\left|a_{L+1} / a_{L}\right| \leqslant \rho^{2 L}\left|a_{1} / a_{0}\right|, \quad L=0,1,2, \ldots,
$$

and

$$
\left|a_{L+k+1} / a_{L+k}\right| \leqslant \rho^{2 k}\left|a_{L+1} / a_{L}\right|, \quad L, k=0,1,2, \ldots
$$

From (7) it follows that

$$
\left|a_{L-k-1} / a_{L-k}\right| \leqslant \rho^{2 k}\left|a_{L-1} / a_{L}\right|, \quad L=1,2, \ldots, k=1,2, \ldots, L-1 .
$$

If we now write

$$
\left|\frac{a_{L+p}}{a_{L}}\right|=\left|\frac{a_{L+p}}{a_{L+p-1}} \cdot \frac{a_{L+p-1}}{a_{L+p-2}} \cdots \cdot \frac{a_{L+1}}{a_{L}}\right|
$$


and estimate each factor once using (8) and once using (9), we obtain

$$
\begin{array}{ll}
\left|a_{L+p} / a_{L}\right| \leqslant \rho^{p(2 L+p-1)}\left|a_{1} / a_{0}\right|^{p}, & L, p=0,1,2, \ldots, \\
\left|a_{L+p} / a_{L}\right| \leqslant \rho^{p(p-1)}\left|a_{L+1} / a_{L}\right|^{p}, & L, p=0,1,2, \ldots
\end{array}
$$

In a similar way, writing

$$
\frac{a_{L-p}}{a_{L}}=\frac{a_{L-p}}{a_{L-p+1}} \cdot \frac{a_{L-p+1}}{a_{L-p+2}} \cdots \cdot \frac{a_{L-1}}{a_{L}}
$$

and using (10), we obtain

$$
\left|a_{L-p} / a_{L}\right| \leqslant \rho^{p(p-1)}\left|a_{L-1} / a_{L}\right|^{p}, \quad L=1,2, \ldots, p=1,2, \ldots, L .
$$

Finally, from (6) it follows that the sequence $\left|a_{j+1} / a_{j}\right|$ is monotonically decreasing so that $\left|a_{k} / a_{k-1}\right|<\left|a_{l} / a_{l-1}\right|$ for $k>l$, or

$$
\left|a_{k} / a_{l}\right|<\left|a_{k-1} / a_{l-1}\right|, \quad k>l .
$$

We now turn to our first lemma, which deals with the determinant $D(L / M)$ (see (4)).

\section{LEMMA 1.}

$$
\left(\frac{1}{2}\right)^{M-1}\left|a_{L}\right|^{M} \leqslant|D(L / M)| \leqslant\left(\frac{3}{2}\right)^{M-1}\left|a_{L}\right|^{M}, \quad L, M=1,2, \ldots
$$

Remark. This lemma was proved in [3]. Since we shall need some ideas from the proof in the sequel, we shall reproduce it, but use a different notation from that in [3].

$$
\text { Proof. Let }
$$

$$
\xi_{L}=\left|a_{L-1} / a_{L+1}\right|^{1 / 2},
$$

and let $\Lambda=\operatorname{diag}\left\{\xi_{L}, \xi_{L}^{2}, \ldots, \xi_{L}^{M}\right\}$. Let $A_{L M}=\left(a_{L-i+j}\right)_{i, j=1}^{M}$ and consider the matrix

$$
\left(b_{i j}\right)=B=a_{L}^{-1} \cdot \Lambda^{-1} \cdot A \cdot \Lambda
$$

that is,

$$
b_{i j}=\frac{a_{L-i+j}}{a_{L}} \xi_{L}^{j-i}, \quad i, j=1, \ldots, M
$$

We shall show that $B$ is a matrix with dominant diagonal, that is,

$$
\boldsymbol{\sigma}=\max _{1 \leqslant i \leqslant M} \sum_{\substack{j=1 \\ j \neq i}}^{M}\left|b_{i j}\right| /\left|b_{i i}\right|<1 .
$$


Indeed, for $1 \leqslant i \leqslant M$, since $\left|b_{i i}\right|=1$,

$$
\begin{aligned}
\sum_{\substack{j=1 \\
j \neq i}}^{M} \frac{\left|b_{i j}\right|}{\left|b_{i i}\right|}= & \sum_{j=1}^{i-1}\left|b_{i j}\right|+\sum_{j=i+1}^{M}\left|b_{i j}\right| \\
& =\sum_{j=1}^{i-1}\left|\frac{a_{L-i+j}}{a_{L}}\right| \cdot\left|\frac{a_{L-1}}{a_{L+1}}\right|^{(j-i) / 2}+\sum_{j=i+1}^{M}\left|\frac{a_{L-i+j}}{a_{L}}\right| \cdot\left|\frac{a_{L-1}}{a_{L+1}}\right|^{(j-i) / 2} \\
= & \sum_{p=1}^{i-1}\left|\frac{a_{L-p}}{a_{L}}\right| \cdot\left|\frac{a_{L-1}}{a_{L+1}}\right|^{-p / 2}+\sum_{p=1}^{M-i}\left|\frac{a_{L+p}}{a_{L}}\right| \cdot\left|\frac{a_{L-1}}{a_{L+1}}\right|^{p / 2}(\text { by }(15),(1) \\
\leqslant & \sum_{p=1}^{i-1} \rho^{p(p-1)}\left|\frac{a_{L-1} a_{L+1}}{a_{L}^{2}}\right|^{p / 2}+\sum_{p=1}^{M-i} \rho^{p(p-1)}\left|\frac{a_{L-1} a_{L+1}}{a_{L}^{2}}\right|^{p / 2} \\
\leqslant & \sum_{p=1}^{i-1} \rho^{p^{2}}+\sum_{p=1}^{M-i} \rho^{p^{2}} \quad(\text { by condition }(2)) \\
< & 2 \sum_{p=1}^{\infty} \rho^{p^{2}}<2 \sum_{p=1}^{\infty} \rho_{0}^{p^{2}}=1,
\end{aligned}
$$

by condition (3) on $\rho$ and by the definition of $\rho_{0}$. So we have

$$
\sigma<2 \sum_{p=1}^{\infty} \rho^{p^{2}}<1
$$

and (18) is proved.

Applying an inequality of Ostrowski [5, formula 8], and noting that the above argument shows that $\sum_{j=1}^{i-1}\left|b_{i j}\right|<\frac{1}{2}$, we obtain,

$$
\left(\frac{1}{2}\right)^{M-1}<\prod_{i=1}^{M-1}\left(1-\sigma \sum_{j=1}^{i-1}\left|b_{i j}\right|\right) \leqslant|\operatorname{det} B| \leqslant \prod_{i=1}^{M-1}\left(1+\sigma \sum_{j=1}^{i-1}\left|b_{i j}\right|\right)<\left(\frac{3}{2}\right)^{M-1} \text {. }
$$

Now, the lemma follows from this inequality and from the relation (16) between $B$ and $\boldsymbol{A}_{L M}$.

LEMMA 2. Let $L, M$ be nonnegative integers and $k \geqslant L+M+1$. Let

$$
A_{L+1, M+1}^{(k)}=\left(\begin{array}{cccc}
a_{L+1} & \cdots & a_{L+M} & a_{k} \\
\vdots & & \vdots & \vdots \\
a_{L-M+2} & \cdots & a_{L+1} & a_{k-M+1} \\
a_{L-M+1} & \cdots & a_{L} & a_{k-M}
\end{array}\right) .
$$

Then

$$
\left|\operatorname{det} A_{L+1, M+1}^{(k)}\right| \leqslant\left(\frac{3}{2}\right)^{M}\left|a_{k-M}\right| \cdot\left|a_{L+1}\right|^{M}
$$


Proof. Multiplying the last column of $A_{L+1, M+1}^{(k)}$ by $a_{L+1} / a_{k-M}$, we obtain the matrix

$$
\tilde{A}_{L+1, M+1}^{(k)}=\left(\begin{array}{cccc}
a_{L+1} & \cdots & a_{L+M} & a_{k} \frac{a_{L+1}}{a_{k-M}} \\
\vdots & & & \vdots \\
a_{L-M+2} & \cdots & a_{L+1} & a_{k-M+1} \frac{a_{L+1}}{a_{k-M}} \\
a_{L-M+1} & \cdots & a_{L} & a_{L+1}
\end{array}\right) .
$$

For $k=L+M+1$, this matrix coincides with $A_{L+1, M+1}$, the matrix whose determinant is $D((L+1) /(M+1))$. For $k>L+M+1, \tilde{A}_{L+1, M+1}^{(k)}$ differs from $A_{L+1, M+1}$ only in the first $M$ elements in the last column, which are less in absolute value than the corresponding elements of $A_{L+1, M+1}$. Indeed, by (14),

$$
\left|\frac{a_{k-i+1}}{a_{k-M}}\right|<\left|\frac{a_{k-i}}{a_{k-M-1}}\right|<\cdots<\left|\frac{a_{L+M+2-i}}{a_{L+1}}\right|, \quad i=1,2, \ldots, M,
$$

so that

$$
\left|a_{k-i+1} \frac{a_{L+1}}{a_{k-M}}\right|<\left|a_{L+M+2-i}\right|, \quad i=1,2, \ldots, M .
$$

Applying the transformation used in the proof of Lemma 1, with $\Lambda=$ $\operatorname{diag}\left\{\xi_{L+1}, \ldots, \xi_{L+1}^{M+1}\right\}$, to $A_{L+1, M+1}$ and to $\tilde{A}_{L+1, M+1}^{(k)}$, we obtain the matrices

$$
B=a_{L+1}^{-1} \Lambda^{-1} A_{L+1, M+1} \Lambda, \quad \tilde{B}=a_{L+1}^{-1} \Lambda^{-1} \tilde{A}_{L+1, M+1}^{(k)} \Lambda .
$$

From the proof of Lemma 1 we know that $B$ has a dominant diagonal and that $\mid$ det $B \mid<\left(\frac{3}{2}\right)^{M}$. But $\tilde{B}$ differs from $B$ only in the first $M$ elements in the last column and these are less in absolute value than the corresponding elements of $B$. It follows that $\tilde{B}$ is also a matrix with dominant diagonal and that $\operatorname{det} \tilde{B}$ satisfies the same estimate: $|\operatorname{det} \tilde{B}|<\left(\frac{3}{2}\right)^{M}$. From this it follows that

$$
\left|\operatorname{det} \tilde{A}_{L+1, M+1}^{(k)}\right|<\left|a_{L+1}\right|^{M+1}\left(\frac{3}{2}\right)^{M} .
$$

Since

$$
\operatorname{det} A_{L+1, M+1}^{(k)}=\frac{a_{k-M}}{a_{L+1}} \operatorname{det} \tilde{A}_{L+1, M+1}^{(k)},
$$

the lemma follows.

LEMMA 3. Let $\tau>0$ satisfy

$$
\tau<L^{-1} \xi_{L}
$$

where $\xi_{L}$ is defined by (15). Suppose that $L$ is sufficiently large, such that $\xi_{L}>1$. Then, for any $M$ complex numbers $c_{1}, \ldots, c_{M}$ that satisfy $\max _{1 \leqslant k \leqslant M}\left|c_{k}\right|=1$, we have

$$
\sum_{j=1}^{M}\left|\sum_{i=1}^{M} c_{i} a_{L-i+j}\right| \tau^{j} \geqslant C\left|a_{L}\right| \xi_{L}^{-(M-1)} \tau^{M}
$$

where $C$ depends only on $\rho$. 
Proof. We first prove that for any $\tau>0$,

$$
\sum_{j=1}^{M}\left|\sum_{i=1}^{M} c_{i} a_{L-i+j}\right| \tau^{j} \geqslant 1 / \max _{1 \leqslant j, k \leqslant M}\left(\tau^{-j}\left|A_{j k}^{-1}\right|\right),
$$

where $A=\left(a_{L-i+j}\right)_{i, j=1}^{M}$ and $A_{j k}^{-1}$ denotes the $(j, k)$ element of $A^{-1}$. Let c denote the row-vector $\left(c_{1}, \ldots, c_{M}\right)$. Since $\mathbf{c}=(\mathbf{c} A) A^{-1}$, we obtain

$$
\left|c_{k}\right|=\left|\sum_{j=1}^{M}\left(\sum_{i=1}^{M} c_{i} a_{L-i+j}\right) A_{j k}^{-1}\right| \leqslant \max _{1 \leqslant j, k \leqslant M}\left|A_{j k}^{-1}\right|\left(\sum_{j=1}^{M}\left|\sum_{i=1}^{M} c_{i} a_{L-i+j}\right|\right) \text {. }
$$

Since $\max _{1 \leqslant k \leqslant M}\left|c_{k}\right|=1$, by assumption, (22) follows for $\tau=1$.

For $\tau \neq 1$, write $\mathbf{c}=\left(\mathbf{c} A_{\tau}\right) A_{\tau}^{-1}$ with $A_{\tau}=A \cdot \operatorname{diag}\left\{\tau, \tau^{2}, \ldots, \tau^{M}\right\}$, and repeat the argument.

REMARK. Estimate (22) is another form of a result of Saff [6, Lemma 2], but the above proof is simpler.

We now estimate $\left|A_{j k}^{-1}\right|$. As was shown in the proof of Lemma 1, the matrix $B=a_{L}^{-1} \Lambda^{-1} A \Lambda\left(\Lambda=\operatorname{diag}\left\{\xi_{L}, \ldots, \xi_{L}^{M}\right\}\right)$ has dominant diagonal. Hence (see Ostrowski [5, formulas (12)-(14)]), the elements of $B^{-1}$ satisfy

$$
\left|B_{j k}^{-1}\right| \leqslant \sigma /\left(1-\sigma^{2}\right), \quad j, k=1, \ldots, M,
$$

where $\sigma$ is defined by (18) and $\sigma<2 \sum_{p=1}^{\infty} \rho^{p^{2}}<1$ by (19). Since the elements of $A^{-1}$ are related to those of $B^{-1}$, by

$$
A_{j k}^{-1}=a_{L}^{-1} \xi_{L}^{j-k} B_{j k}^{-1}, \quad j, k=1, \ldots, M,
$$

we obtain

$$
\left|A_{j k}^{-1}\right| \leqslant C_{1}\left|a_{L}\right|^{-1} \xi_{L}^{j-k}, \quad j, k=1, \ldots, M,
$$

where $C_{1}$ depends only on $\rho$. Hence,

$$
\begin{aligned}
\max _{1 \leqslant j, k \leqslant M} \tau^{-j}\left|A_{j k}^{-1}\right| & \leqslant C_{1}\left|a_{L}\right|^{-1} \max _{1 \leqslant j, k \leqslant M} \tau^{-j} \xi_{L}^{j-k} \\
& =C_{1}\left|a_{L}\right|^{-1} \max _{1 \leqslant j \leqslant M} \tau^{-j} \xi_{L}^{j-1} \quad\left(\text { since } \xi_{L}>1\right) \\
& =C_{1}\left|a_{L}\right|^{-1} \tau^{-M} \xi_{L}^{M-1},
\end{aligned}
$$

since $\tau / \xi_{L}<1 / L<1$ by the assumption (21). Substituting the last estimate in (22), we obtain the assertion of the lemma.

We now establish the properties of the Padé approximants to functions $f$ that satisfy (1)-(3).

LEMMA 4. For each fixed $r>0$, there exists a positive integer $L_{0}=L_{0}(r)$, such that for $L \geqslant L_{0}$ and $M=1,2,3, \ldots$,

$$
\left(1-2 r / \xi_{L}\right)^{M} \leqslant\left|Q_{L M}(z)\right| \leqslant\left(1+2 r / \xi_{L}\right)^{M}, \quad|z| \leqslant r,
$$

where $\xi_{L}$ is defined by (15). 
Proof. Let

$$
Q_{L M}(z)=\prod_{j=1}^{M}\left(1-\frac{z}{z_{L M j}}\right),
$$

where $z_{L M j}, j=1, \ldots, M$, are the zeros of $Q_{L M}$ (if some of them lie at $\infty$, we replace the corresponding factor by 1). It follows from (11) in [3] that $z_{L M j}$ satisfy $\left|z_{L M j}\right|>\frac{1}{2} \xi_{L}$ (in the notation of [3] the bound is $\frac{1}{2}\left(q_{L} q_{L+1}\right)^{1 / 2}$ with $q_{j}=\left|a_{j-1} / a_{j}\right|$, $j=1,2,3, \ldots)$. The lemma follows from this estimate since $\xi_{L} \rightarrow \infty$ as $L \rightarrow \infty$.

LEMma 5. Let $f$ satisfy (1)-(3) and let $[L / M]=P_{L M} / Q_{L M}$ be the $L, M$ Padé approximant to $f$. Then,

$$
\|f-[L / M]\|_{1} \leqslant C \cdot 3^{M}\left|a_{L+1}\right| \cdot\left|\frac{a_{L+1}}{a_{L}}\right|^{M} /\left(1-\frac{2}{\xi_{L}}\right)^{M},
$$

where $C$ depends only on $f$.

Proof. By equation (1.1) in Arms and Edrei [1, p. 8] and using our notation (20), we obtain

$$
\left(f Q_{L M}-P_{L M}\right)(z)=\frac{(-1)^{M}}{D(L / M)} \sum_{k=L+M+1}^{\infty}\left(\operatorname{det} A_{L+1, M+1}^{(k)}\right) z^{k} .
$$

So, for $|z| \leqslant 1$,

$$
\begin{aligned}
& \left|\left(f Q_{L M}-P_{L M}\right)(z)\right| \leqslant \frac{1}{|D(L / M)|} \sum_{k=L+M+1}^{\infty}\left|\operatorname{det} A_{L+1, M+1}^{(k)}\right| \\
& \quad \leqslant \frac{1}{|D(L / M)|} \cdot\left(\frac{3}{2}\right)^{M} \cdot\left|a_{L+1}\right|^{M} \sum_{k=L+M+1}^{\infty}\left|a_{k-M}\right| \text { (by Lemma 2). }
\end{aligned}
$$

Estimating $|D(L / M)|$ from below by Lemma 1 and taking into account that $\sum_{k=L+M+1}^{\infty}\left|a_{k-M}\right| \leqslant C\left|a_{L+1}\right|$, where $C$ depends on $f$ (by (8), for example), we obtain

$$
\left|\left(f Q_{L M}-P_{L M}\right)(z)\right| \leqslant C 3^{M}\left|a_{L+1}\right| \cdot\left|\frac{a_{L+1}}{a_{L}}\right|^{M}, \quad|z| \leqslant 1 .
$$

Dividing by $Q_{L M}(z)$ and using Lemma 4, we obtain the required estimate.

LEMMA 6. Let

$$
0<c<c_{0}=2\left|\log \rho_{0}\right| / \log 3=1.42995 \ldots
$$

Let $\varepsilon_{L M}$ be defined by (5). Then for any sequence of nonnegative integers $\left\{M_{L}\right\}_{L=1}^{\infty}$ that satisfies $M_{L} \leqslant c L, L=1,2,3, \ldots$,

$$
\lim _{L \rightarrow \infty} \frac{f(z)-\left[L / M_{L}\right](z)}{(-1)^{M} \varepsilon_{L M_{L .}} z^{L+M_{I .}+1}}=1
$$

uniformly on compact subsets of $\mathbf{C}$. 
Proof. From the formula (23) and the fact that

$$
\operatorname{det} A_{L+1, M+1}^{(L+M+1)}=D((L+1) /(M+1))
$$

by (20), we obtain

$$
\begin{aligned}
& \frac{\left(f Q_{L M}-P_{L M}\right)(z)}{(-1)^{M} D((L+1) /(M+1)) z^{L+M+1} / D(L / M)} \\
& \quad=1+\sum_{k=L+M+2}^{\infty} \frac{1}{D((L+1) /(M+1))}\left(\operatorname{det} A_{L+1, M+1}^{(k)}\right) z^{k-L-M-1} .
\end{aligned}
$$

The absolute value of the sum is estimated by Lemmas 1 and 2 :

$$
\begin{aligned}
|\Sigma| & \leqslant \sum_{k=L+M+2}^{\infty} \frac{\left|a_{k-M}\right| \cdot\left|a_{L+1}\right|^{M}}{\left(\frac{1}{2}\right)^{M}\left|a_{L+1}\right|^{M+1}} \cdot\left(\frac{3}{2}\right)^{M} \cdot|z|^{k-L-M-1} \\
& \leqslant 3^{M}\left|\frac{a_{L+2}}{a_{L+1}}\right| \sum_{k=L+M+2}^{\infty}\left|\frac{a_{k-M}}{a_{L+2}}\right| \cdot|z|^{k-L-M-1} \\
& =3^{M}\left|\frac{a_{L+2}}{a_{L+1}}\right| \sum_{p=0}^{\infty}\left|\frac{a_{L+2+p}}{a_{L+2}}\right| \cdot|z|^{p+1} \\
& \leqslant 3^{M} \rho^{2 L}\left\{\rho^{2}\left|\frac{a_{1}}{a_{0}}\right| \sum_{p=0}^{\infty}|z|\left(|z| \rho^{2 L+p+3} \cdot\left|\frac{a_{1}}{a_{0}}\right|\right)^{p}\right\} \quad \text { (by (11), (8)). }
\end{aligned}
$$

If $M=M_{L} \leqslant c L$, we have $\left(3^{M} \rho^{2 L}\right) \leqslant\left(3^{c} \rho^{2}\right)^{L} \rightarrow 0$ as $L \rightarrow \infty$, since $3^{c} \rho^{2}<1$ by choice of $c$. The term in the braces in (25) is obviously uniformly bounded on each compact subset of $\mathbf{C}$. Hence, the sum on the right-hand side of (24) tends to zero uniformly on compact subsets of $\mathbf{C}$ and we obtain

$$
\frac{\left(f Q_{L M}-P_{L M}\right)(z)}{(-1)^{M} \varepsilon_{L M} z^{L+M+1}}=1+o(1) \quad \text { as } L \rightarrow \infty \text { and } M=M_{L} \leqslant c L .
$$

From (15) and (8), it follows that $1 / \xi_{L} \leqslant C \rho^{2 L}, L=1,2,3, \ldots$ Then, from Lemma 4, it follows that for $M=M_{L} \leqslant c L, Q_{L M}(z) \rightarrow 1$ uniformly on compact subsets of C. Dividing (26) by $Q_{L M}(z)$, we obtain the assertion of the lemma.

3. Proofs of Theorems 1,2. As was mentioned in the introduction, the properties of the Padé table that were established in $\$ 2$ enable us to adapt Saff's arguments to our case. However, for the sake of completeness, we shall give a fairly full proof of Theorem 1.

Proof OF THEOREM 1. Let $f$ satisfy (1)-(3) and let $W_{L M}$ be a rational function of best approximation to $f$ in the uniform norm on $|z| \leqslant r$. Since condition (2) is invariant under the transformation $f(z) \rightarrow f(r z)$, it is sufficient to consider the case $r=1$. Let $L, M$ be fixed and assume that $W_{L M}$ has a pole $1 / \alpha_{L M}\left(\left|\alpha_{L M}\right|<1\right)$ in the disk $|z| \leqslant \tau / 2(\tau>2)$. Assuming $L$ sufficiently large, we shall obtain a lower bound for $\tau$. Write

$$
W_{L M}(z)=p_{L M}(z) /\left[\left(1-\alpha_{L M} z\right) q_{L M}(z)\right]
$$


where $p_{i, M}$ is a polynomial of degree $\leqslant L$ and $q_{I M}$ is a polynomial of the form

$$
q_{L . M}(z)=\sum_{i=1}^{M} c_{i} z^{i-1}
$$

which is normalized so that $\max _{1 \leqslant i \leqslant M}\left|c_{i}\right|=1$. Note that then

$$
\left|q_{L M}(z)\right| \leqslant M, \quad|z| \leqslant 1 .
$$

By the extremal property of $W_{L M}$ we have

$$
\begin{aligned}
\left|W_{L M}(z)-[L / M](z)\right| & \leqslant\left|W_{L M}(z)-f(z)\right|+|f(z)-[L / M](z)| \\
& \leqslant 2\|f-[L / M]\|_{1}, \quad|z| \leqslant 1,
\end{aligned}
$$

where $[L / M]=P_{L M} / Q_{L M}$ is the Padé approximant of type $(L, M)$ for $f$. Now, (27), (29) and the estimate from above for $Q_{L M}$ (Lemma 4) imply

$$
\begin{aligned}
& \left|p_{L . M}(z) Q_{L . M}(z)-P_{L M}(z)\left(1-\alpha_{L M} z\right) q_{L . M}(z)\right| \\
& \quad \leqslant 4 M\|f-[L / M]\|_{1}\left(1+2 / \xi_{I .}\right)^{M}, \quad|z| \leqslant 1 .
\end{aligned}
$$

From this we deduce by Bernstein's Lemma (Walsh [10, p. 77]) that

$$
\begin{aligned}
& \left|p_{L M}(z) Q_{L M}(z)-P_{L M}(z)\left(1-\alpha_{I M} z\right) q_{L M}(z)\right| \\
& \quad \leqslant \tau^{L+M} \cdot 4 M \cdot\|f-[L / M]\|_{1}\left(1+2 / \xi_{L}\right)^{M}, \quad|z| \leqslant \tau .
\end{aligned}
$$

For $L$ sufficiently large, $\left|Q_{L M}\right|$ is bounded from below on $|z| \leqslant \tau$ by Lemma 4 . Hence,

$$
\begin{aligned}
& \left|\left(1-\alpha_{L M} z\right) q_{L M}(z) \cdot[L / M](z)-p_{L M}(z)\right| \\
& \quad \leqslant \tau^{L+M} \cdot 4 M \cdot\|f-[L / M]\|_{1} \cdot\left(\left(1+2 / \xi_{L}\right) /\left(1-2 \tau / \xi_{L}\right)\right)^{M} .
\end{aligned}
$$

Since this inequality holds for $z=1 / \alpha_{L M}$, it follows that

$$
\begin{gathered}
\left|\left(1-\alpha_{L M} z\right) q_{L M}(z)[L / M](z)-p_{I, M}(z)+p_{L M}\left(1 / \alpha_{L M}\right)\right| \\
\leqslant 2 \times \text { (right-hand member of }(30)), \quad|z| \leqslant \tau .
\end{gathered}
$$

Consequently, noting that $\left|1-\alpha_{L M} z\right| \geqslant(2 / \tau) \tau-1=1$ for $|z| \leqslant \tau$, we obtain

$$
\begin{aligned}
& \left\|q_{L, M}[L / M]-\pi_{L M}\right\|_{\tau} \\
& \quad \leqslant \tau^{L+M} \cdot 8 M \cdot\|f-[L / M]\|_{1} \cdot\left(\left(1+2 / \xi_{L}\right) /\left(1-2 \tau / \xi_{L}\right)\right)^{M},
\end{aligned}
$$

where $\pi_{L M}(z)=\left[p_{L M}(z)-p_{L M}\left(1 / \alpha_{L M}\right)\right] /\left(1-\alpha_{L M} z\right)$ is a polynomial of degree $\leqslant L-1$.

Estimating $\|f-[L / M]\|_{1}$ by Lemma 5 and assuming that $\tau<L^{-1} \xi_{L}$, we obtain, from (31),

$$
\left\|q_{L M}[L / M]-\pi_{L M}\right\|_{\tau} \leqslant \tau^{L+M} C M 3^{M}\left|a_{L+1}\right| \cdot\left|a_{L+1} / a_{L}\right|^{M}\left(1+\delta_{L}\right)^{M},
$$
where $\delta_{I} \rightarrow 0$ as $L \rightarrow \infty$.

We now obtain a lower bound for the left-hand member of (32). In a neighbourhood of 0 , let

$$
q_{L M}(z)[L / M](z)-\pi_{L M}(z)=\sum_{k=0}^{\infty} d_{k} z^{k}
$$


Since $\operatorname{deg} \pi_{L M} \leqslant L-1$, and taking into account (28) and the definition of $[L / M]$, we obtain

$$
d_{k}=\sum_{i=1}^{M} a_{k-i+1} c_{i}, \quad k=L, L+1, \ldots, L+M-1 .
$$

Now, it follows from (33) that

$$
\begin{aligned}
\left\|q_{L M}[L / M]-\pi_{L M}\right\|_{\tau}^{2} & \geqslant(2 \pi \tau)^{-1} \int_{|z|=\tau}\left|\sum_{k=0}^{\infty} d_{k} z^{k}\right|^{2}|d z| \\
& \geqslant \sum_{k=L}^{L+M-1}\left|d_{k}\right|^{2} \tau^{2 k} \geqslant \frac{1}{M}\left(\sum_{k=L}^{L+M-1}\left|d_{k}\right| \tau^{k}\right)^{2}
\end{aligned}
$$

by the Cauchy-Schwarz inequality. Hence, by (34),

$$
\begin{aligned}
\left\|q_{L M}\left[\frac{L}{M}\right]-\pi_{L M}\right\|_{\tau} & \geqslant \frac{1}{\sqrt{M}} \sum_{k=L}^{L+M-1}\left|\sum_{i=1}^{M} c_{i} a_{k-i+1}\right| \tau^{k} \\
& =\frac{\tau^{L-1}}{\sqrt{M}} \sum_{j=1}^{M}\left|\sum_{i=1}^{M} c_{i} a_{L-i+j}\right| \tau^{j} \\
& \geqslant \tau^{L+M-1} \cdot \frac{C}{\sqrt{M}}\left|a_{L}\right| \xi_{L}^{-(M-1)}
\end{aligned}
$$

by Lemma 3 (remember that we assumed that $\tau<L^{-1} \xi_{L}$ ). Now (32), (35) and the definition (15) of $\xi_{L}$ yield

$$
\begin{aligned}
\tau \geqslant & C \cdot M^{-3 / 2} 3^{-M}\left|a_{L} / a_{L+1}\right| \cdot\left|a_{L-1} / a_{L+1}\right|^{1 / 2} \\
& \cdot\left|a_{L}^{2} /\left(a_{L-1} a_{L+1}\right)\right|^{M / 2} \cdot\left(1+\delta_{L}\right)^{-M},
\end{aligned}
$$

provided $\tau<L^{-1}\left|a_{L-1} / a_{L+1}\right|^{1 / 2}$. Using (2), (8), we finally obtain,

$$
\tau \geqslant C \cdot \min \left\{L^{-1} \rho^{-2 L}, M^{-3 / 2}(3 \rho)^{-M} \rho^{-4 L}\left(1+\delta_{L}\right)^{-M}\right\},
$$

where $C$ depends only on $f$ and $\delta_{L}=\delta_{L}(f) \rightarrow 0$ as $L \rightarrow \infty$.

It is easily verified that for $\rho<\frac{1}{3}$ the right-hand member of (36) tends to infinity as $L \rightarrow \infty$ and $M=M_{L}$ arbitrary, and that for $\frac{1}{3} \leqslant \rho<\rho_{0}=0.4559 \ldots$ the same is true provided $M=M_{L}$ satisfies $M_{L} \leqslant c L, L=1,2, \ldots$, where $c<c_{1}=$ $4\left|\log \rho_{0}\right| / \log \left(3 \rho_{0}\right)=10.0339 \ldots$ So, the poles of $W_{L M_{t}}$ tend to infinity as $L \rightarrow \infty$. It is well known (see Walsh [10, Corollary 5, p. 234]) that this fact implies the uniform convergence of $W_{L M_{l}}$ to $f$ on compact subsets of $\mathbf{C}$. Theorem 1 is proved.

Proof of Theorem 2. Let $c_{0}=1.42995 \ldots$ be the constant that appears in Lemma 6. Let $\left\{M_{L}\right\}_{L=1}^{\infty}$ be any sequence of integers that satisfies $M_{L} \leqslant c L$, $L=1,2, \ldots$, where $c<c_{0}$. Then Lemma 6 holds.

Furthermore, from (36) it follows that the poles of $W_{L M_{L}}$ lie outside the disk $|z|<T^{L}(T>1)$. Hence, if we normalize the denominator $q_{L M_{l} \text {. }}$ of $W_{L M_{l}}$ by $q_{L M_{l}}(0)=1$, we obtain

$$
\lim _{L \rightarrow \infty} q_{L M_{L}}(z)=1, \quad M_{L} \leqslant c L, L=1,2, \ldots,
$$


uniformly on compact subsets of $\mathbf{C}$. This fact, together with Lemma 6, enables us to use Saff's method of the proof of Theorems B and C without any changes, and Theorem 2 follows.

We also note that part (i) of Theorem 2 follows immediately from Lemma 6 and Rouché's theorem (cf. the proof in Trefethen [9]).

REMARKS. 1. It follows from the proof of Theorem 1, that this theorem is valid not only for the "best" rational functions $W_{L M}$ but for any sequence of rational functions $R_{L M}$ of respective types $(L, M)$ that satisfy for some $r>0:\left\|f-R_{L M}\right\|_{r}$ $\leqslant C\|f-[L / M]\|_{r}$ (cf. the remark in Saff [7, p. 193]).

2. From the previous remark follows via the lemma of Walsh [10, p. 101], that Theorem 1 is also true for the case of best rational approximation in the $L_{p}$-norm, $p>0$, on $|z|=r$.

3. In the proof of Theorem 1 we assumed for simplicity of writing that $r=1$. With a little more effort a stronger result can be obtained, namely:

THEOREM 1'. Let $f$ satisfy (1)-(3) and let $W_{L M}(f ; r)$ denote a rational function of type $(L, M)$ of best approximation to $f$ in the uniform norm on $|z| \leqslant r$. Let $\beta_{L M}(f ; r)$ denote the pole of $W_{L M}(f ; r)$, nearest to the origin. Then for any sequence of indices $\left\{M_{L}\right\}_{I=1}^{\infty}$ such that $M_{L} \leqslant 10 L, L=1,2,3, \ldots$, the following holds:

$$
\inf _{r>0}\left|\beta_{L, M_{l}}(f ; r)\right| \rightarrow \infty \quad \text { as } L \rightarrow \infty
$$

4. It seems likely that the constant $\rho_{0}$ is sharp in the sense that for every $\rho_{1}>\rho_{0}$ there exists an entire function which satisfies (1) and (2) with $\rho=\rho_{1}$ and such that the set of poles of the functions $W_{I M_{l}}(f ; r), M_{I} \leqslant 10 L, L=1,2,3, \ldots$, has a finite limit point.

Although we cannot prove this fact, we can motivate it by proving that for $\rho=\rho_{1}>\rho_{0}$, the sharpened version of Theorem 1 (Theorem $1^{\prime}$ ) definitely fails. Indeed, according to a result of Walsh [11], if $D(L / M) \neq 0$ for some $L, M$, then as $r \rightarrow 0$, the poles of $W_{L M}(f ; r)$ tend to the poles of $[L / M]$. So, to contradict Theorem $1^{\prime}$ it suffices to prove that for any $\rho_{1}, \rho_{1}>\rho_{0}$, there exists an entire function which satisfies (1) and (2) with $\rho=\rho_{1}$, and such that some sequence $\left[L / M_{L}\right]$ of its Padé approximations has a finite limit point. This was proved in [4] even for the case $M_{L}=$ const.

\section{REFERENCES}

1. R. J. Arms and A. Edrei, The Pade tables and continued fractions generated by totally positive sequences, Mathematical Essays, Dedicated to A. J. Macintyre, Ohio Univ. Press, 1970, pp. 1-21.

2. D. Braess, On the conjecture of Meinardus on rational approximation to $e^{x}$. II, J. Approx. Theory 40 (1984), 375-379.

3. D. S. Lubinsky, Padé tables of a class of entire functions, Proc. Amer. Math. Soc. 94 (1985), 399-405.

4. Pade tables of entire functions of very slow and smooth growth, Constructive Approx. (to appear).

5. A. Ostrowski, Note on bounds for determinants with dominant principal diagonal, Proc. Amer. Math. Soc. 3 (1952), 26-30. 
6. E. B. Saff, The convergence of rational functions of best approximation to the exponential function, Trans. Amer. Math. Soc. 153 (1971), 483-493.

7. The convergence of rational functions of best approximation to the exponential function. II, Proc. Amer. Math. Soc. 32 (1972), 187-194.

8. , On the degree of best rational approximation to the exponential function, J. Approx. Theory 9 (1973), 97-101.

9. L. N. Trefethen, The asymptotic accuracy of rational best approximation to $e^{x}$ on a disk, J. Approx. Theory 40 (1984), 380-383.

10. J. L. Walsh, Interpolation and approximation by rational functions in the complex domain, 2 nd ed., Amer. Math. Soc. Colloq. Publ., vol. 20, Amer. Math. Soc., Providence, R. I., 1956.

11. , Pade approximations as limits of rational functions of best approximation, J. Math. Mech. 13 (1964), 305-312.

Department of Mathematics, Everyman's University, P.O. Box 39328, Tel Aviv, Israel

National Research Institute for Mathematical Sciences of the CSIR, P. O. Box 395, Pretoria 0001, Republic of South Africa 